\title{
Optimization of Partly Conflicting Goals in Complex Resource Planning \\ Rudolf Felix
}

\author{
F/L/S Fuzzy Logik Systeme GmbH, Joseph-von-Fraunhofer Straße 20, 44227 Dortmund \\ Tel. +49 231 9700921, Fax. +49 231 9700929, Email: felix@ fuzzy.de
}

\begin{abstract}
Many optimization models are limited with respect to the management of inconsistency between the optimization goals. As consequence, these models in many cases achieve results that are optimal for the model but not for the problem. In real world problems the optimization goals are usually partly conflicting. Independence of goals as precondition usually does not hold and utility functions based on weighted sums only do not really help. In this paper we describe an application of an optimization model based on interactions between goals to workforce management. Workforce management is a relevant representative multi-goal real world resource planning optimization problem.
\end{abstract}

Keywords: Real world optimization problem, fuzzy interactions between goals, resource planning, conflicting goals

\section{Introduction}

In [6] it is already discussed that many decision making approaches [10], [11], [12], [13], [14] are limited with respect to the management of inconsistency between decision goals or do not consider the issue explicitly. A similar category of limitation is observed with respect to optimization goals, too. The consequence of this limitation is that the models are rather not applicable for real world problems when the exactness of the results must optimize the let's say last ten percent of the optimization potential.

The limitations result from the fact that different aggregation approaches are suitable for different types of aggregation situations such as merging uncertain information or aggregation of preference profiles [2]. In real world optimization a mixture of types of aggregation is required from optimization to optimization. Roughly speaking, depending on the optimization situation the reliability of information sources is a result of conflict analysis between the goals. Also depending on the optimization situation preference rankings vary from state to state of the decision situation. Therefore aggregation for decision under uncertainty comes into play. Additionally, if different rankings of the decision alternatives are individually derived from the point of view of each goal, aspects of aggregation in the sense of multicriteria decision making arise. All these aspects have to be considered simultaneously in an optimization framework. In optimization the suitability of different types of aggregation depends more on the question to which extent there are trade-offs or redundancies or even synergies between goals. Therefore it is hardly possible to find a single aggregation approach that integrates all the mentioned requirements simultaneously. In real world optimization many times it has to be calculated dynamically from decision situation to decision situation how and to which extent the goals interact. Therefore automated calculation of the type of interaction is more important compared to rather static decisions in a classical sense where the structure of the decision situation may be a priority well understood.

In a former work [7] it is discussed in which way a decision making approach based on interactions between goals (DMIG) is adapted to the aggregation of inconsistent preference information in complex decision situations [8]. There it is shown that decision making based on interactions between decision goals is better applicable to the calculation of complex decisions compared to (monocentric) concepts based on utility functions and weighted sums. Since in DMIG the interactions between the goals are calculated first and after this calculation the way of aggregation is selected it is not pre-assumed that the goals are redundant or in conflict per se. This aspect is different compared to other aggregation approaches.

It turned out that when using DMIG the complexity of the aggregation process is not higher than polynomial per decision. Since the model has been successfully applied to many real world problems [5] it clearly helped to manage many complex aggregation processes.

In this paper we show a new real world application in a context of partly inconsistent optimization goals in complex resource planning.

The application is the so called workforce management optimization problem. In workforce management a set of tasks have to be mapped to teams of employees, the so called workforce. More precisely the problem is how to attach tasks to resources in a way that all tasks are finished in time, the capacities of the resources are optimally used and a set of additional limitations are optimally exploited. In this sense workforce management is representative for many real world resource planning problems. The limitations are understood as key performance indicators (KPIs) that express the quality of a solution.

In this paper we show that an optimization heuristic based on the evaluation of relationships between decision goals helps to solve this representative problem in an efficient way. The optimization with multiple goals 
is understood as an iteration process of multiple goals decisions.

In the subsequent sections first for better readability of the paper, we repeat the description of the decision making approach based on interactions between decision goals (that has initially been developed at the beginning of the nineties, see also [6]) and show how it is used for optimization. Then we describe the problem of workforce management as an optimization problem. After this we describe, based on some examples of optimization goals, their modeling in terms of the DMIG. Finally, we discuss the results and conclude with some closing remarks.

\section{Decision making based on interactions between goals}

In the following it is shown how an explicit modeling of interactions between decision goals that are defined as fuzzy sets of decision alternatives helps to manage the complexity of the decision making and aggregation process. This modeling of the decision making and aggregation process significantly differs from the related approaches and the way they manage complex decision situations. First the notion of positive and negative impact sets is introduced. Then different types of interaction between goals are defined. After this it is shown how interactions between goals are used in order to aggregate pairs of goals to the so-called local decision sets. Then it is described how the local decision sets are used for the aggregation of a final decision set. The complexity of the different steps is discussed.

\subsection{Positive and negative impact sets}

Before we define interactions between goals as fuzzy relations, we introduce the notion of the positive impact set and the negative impact set of a goal. A more detailed discussion can be found in [3],[4] and [5].

Def. 1a) Let A be a non-empty and finite set of decision alternatives, $\mathrm{G}$ a non-empty and finite set of goals, $\mathrm{A} \cap \mathrm{G}=\varnothing, a \in A, g \in G, \delta \in(0,1]$.

For each goal $g$ we define the two fuzzy sets $S_{g}$ and $D_{g}$ each from $\mathrm{A}$ into $[0,1]$ by:

1. Positive impact function of the goal $g: S_{g}(a):=\delta$, if a affects g positively with degree $\delta, \mathrm{S}_{\mathrm{g}}(\mathrm{a}):=0$ else

2. Negative impact function of the goal $g: D_{g}(a):=\delta$, if a affects $g$ negatively with degree $\delta, D_{g}(a):=0$ else.

Def. $1 b)$ Let $\mathrm{S}_{\mathrm{g}}$ and $\mathrm{D}_{\mathrm{g}}$ be defined as in Def. 1a). $S_{g}$ is called the positive impact set of $g$ and $D_{g}$ the negative impact set of $\mathrm{g}$.

The set $\mathrm{S}_{\mathrm{g}}$ contains alternatives with a positive impact on the goal $\mathrm{g}$ and $\delta$ is the degree of the positive impact. In other words $S_{g}$ could also be called the set of decision alternatives that are good for the goal g. The set $\mathrm{D}_{\mathrm{g}}$ contains alternatives with a negative impact on the goal $\mathrm{g}$ and $\delta$ is the degree of the negative impact. In other words $\mathrm{D}_{\mathrm{g}}$ could also be called the set of decision alternatives that are bad for the goal $\mathrm{g}$.

\subsection{Interactions between goals}

Let $\mathrm{P}(\mathrm{A})$ be the set of all fuzzy subsets of $\mathrm{A}$. Let $X, Y \in P(A), x$ and $y$ the membership functions of $X$ and $\mathrm{Y}$ respectively. Assume now that we have a binary fuzzy inclusion I: $\boldsymbol{P}(A) \times \boldsymbol{P}(A) \rightarrow[0,1]$ and a fuzzy noninclusion $\quad \mathrm{N}: \mathcal{P}(A) \times \mathcal{P}(A) \rightarrow[0,1]$, such that $\mathrm{N}(X, Y):=1-\mathrm{I}(X, Y)$. In such a case the degree of inclusions and non-inclusions between the impact sets of two goals indicates the degree of the existence of interaction between these two goals. The higher the degree of inclusion between the positive impact sets of two goals, the more cooperative the interaction between them. The higher the degree of inclusion between the positive impact set of one goal and the negative impact set of the second, the more competitive the interaction. The non-inclusions are evaluated in a similar way. The higher the degree of non-inclusion between the positive impact sets of two goals, the less cooperative the interaction between them. The higher the degree of noninclusion between the positive impact set of one goal and the negative impact set of the second, the less competitive the relationship. The pair $(\mathrm{Sg}, \mathrm{Dg})$ represents the whole known impact of alternatives on the goal g. Then $\mathrm{Sg}$ is the fuzzy set of alternatives which satisfy the goal g. Dg is the fuzzy set of alternatives, which are rather not recommendable from the point of view of satisfying the goal $\mathrm{g}$.

Based on the inclusion and non-inclusion between the impact sets of the goals as described above, 8 basic fuzzy types of interaction between goals are defined. The different types of interaction describe the spectrum from a high confluence between goals (analogy) to a strict competition (trade-off) [3]

Def. 2) Let $\mathrm{S}_{\mathrm{g}_{1}}, \mathrm{D}_{\mathrm{g}_{1}}, \mathrm{~S}_{\mathrm{g}_{2}}$ and $\mathrm{D}_{\mathrm{g}_{2}}$ be fuzzy sets given by the corresponding membership functions as defined in Def. 1). For simplicity we write $S_{1}$ instead of $\mathrm{S}_{\mathrm{g}_{1}}$ etc.. Let $\mathrm{g}_{1}, \mathrm{~g}_{2} \in \mathrm{G}$ where $\mathrm{G}$ is a set of goals. $T$ is a t-norm.

The fuzzy types of interaction between two goals are defined as relations which are fuzzy subsets of $G \times G$ as follows:

1. $\mathrm{g}_{1}$ is independent of $\mathrm{g}_{2}:<=>$

$$
T\left(\mathrm{~N}\left(S_{1}, S_{2}\right), \mathrm{N}\left(S_{1}, D_{2}\right), \mathrm{N}\left(S_{2}, D_{1}\right), \mathrm{N}\left(D_{1}, D_{2}\right)\right)
$$

2. $\mathrm{g}_{1}$ assists $\mathrm{g}_{2}:<=>T\left(\mathrm{I}\left(S_{1} S_{2}\right), \mathrm{N}\left(S_{1}, D_{2}\right)\right)$

3. $\mathrm{g}_{1}$ cooperates with $\mathrm{g}_{2}:<=>$

$$
T\left(\mathrm{I}\left(S_{1}, S_{2}\right), \mathrm{N}\left(S_{1}, D_{2}\right), \mathrm{N}\left(S_{2}, D_{1}\right)\right)
$$

4. $\mathrm{g}_{1}$ is analogous to $\mathrm{g}_{2}:<=>$

$$
T\left(\mathrm{I}\left(s_{1}, S_{2}\right), \mathrm{N}\left(s_{1}, D_{2}\right), \mathrm{N}\left(s_{2}, D_{1}\right), \mathrm{I}\left(D_{1}, D_{2}\right)\right)
$$

5. $\mathrm{g}_{1}$ hinders $\mathrm{g}_{2}:<=>T\left(\mathrm{~N}\left(S_{1}, S_{2}\right), \mathrm{I}\left(S_{1}, D_{2}\right)\right)$ 
6. $\mathrm{g}_{1}$ competes with $\mathrm{g}_{2}:<=>$

$$
T\left(\mathrm{~N}\left(S_{1}, S_{2}\right), \mathrm{I}\left(S_{1}, D_{2}\right), \mathrm{I}\left(S_{2}, D_{1}\right)\right)
$$

7. $\mathrm{g}_{1}$ is in trade-off to $\mathrm{g}_{2}:<=>$

$$
T\left(\mathrm{~N}\left(S_{1}, S_{2}\right), \mathrm{I}\left(S_{1}, D_{2}\right), \mathrm{I}\left(S_{2}, D_{1}\right), \mathrm{N}\left(D_{1}, D_{2}\right)\right)
$$

8. $\mathrm{g}_{1}$ is unspecified dependent from $\mathrm{g}_{2}:<=>$

$$
T\left(\mathrm{I}\left(S_{1}, S_{2}\right), \mathrm{I}\left(S_{1}, D_{2}\right), \mathrm{I}\left(S_{2}, D_{1}\right), \mathrm{I}\left(D_{1}, D_{2}\right)\right)
$$

The interactions between goals are crucial for an adequate orientation during the decision making process because they reflect the way the goals depend on each other and describe the pros and cons of the decision alternatives with respect to the goals. For example, for cooperative goals a conjunctive aggregation is appropriate. If the goals are rather competitive, then an aggregation based on an exclusive disjunction is appropriate.

Please note that the complexity of the calculation of every type of interaction between two goals in terms of the $\mathrm{O}$ calculus is $\mathrm{O}(\operatorname{card}(\mathrm{A}) * \operatorname{card}(\mathrm{A}))$ [6].

Please note also that the relationships are not symmetrical in general because the degree of the relationship depends on the (fuzzy) cardinality of both the positive and the negative impact sets that is not necessarily equal for two different goals.

For instance, assume that we have two companies that cooperate in a project. Assume that the first one is rather small and the second rather a global player. From the point of view of the small company the project may be the only one the company has. From the point of view of the global player the project with this small company is maybe one among thousands. In such a constellation it should be clear that the cooperation relation between both companies is not a symmetric one despite of the linguistically expected symmetry.

\subsection{Two goals aggregation based on the type of their interaction}

The assumption, that cooperative types of interaction between goals imply conjunctive aggregation and conflicting types of interaction between goals rather lead to exclusive disjunctive aggregation, is easy to accept from the intuitive point of view. It is also easy to accept that in case of independent or unspecified dependent goals a disjunctive aggregation is appropriate. For a more detailed formal discussion see for instance [3], [4]. Knowing the type of interaction between two goals means to recognize for which goals rather a conjunctive aggregation is appropriate and for which goals rather a disjunctive or even exclusively disjunctive aggregation is appropriate. This knowledge then in connection with information about goal priorities is used in order to apply interaction-dependent aggregation policies which describe the way of aggregation for each type of interaction. The aggregation policies define which kind of aggregation operation is the appropriate one for each pair of goals. The aggregation of two goals gi and $\mathrm{gj}$ leads to the so-called local decision set Li,j.. For each pair of goals there is a local decision set $\mathrm{Li}, \mathrm{j}$. $\in \mathrm{P}(\mathrm{A})$, where $A$ is the set of decision alternatives (see Def 1 a)) and $\mathrm{P}(\mathrm{A})$ the power set upon A. For conflicting goals, for instance, the following aggregation policy which deduces the appropriate decision set is given:

if $\left(g_{1}\right.$ is in trade-off to $\left.g_{2}\right)$ and $\left(g_{1}\right.$ is slightly more important than $g_{2}$ ) then $L_{1,2}:=S_{1} / D_{2}$.

In case of very similar goals (analogous or cooperative goals) the priority information even is not necessary: if $\left(g_{1}\right.$ cooperates with $\left.g_{2}\right)$ then $L_{1,2}:=S_{1} \cap S_{2}$ because $S_{1} \cap S_{2}$ surely satisfies both goals. if $\left(g_{1}\right.$ is independent of $\left.g_{2}\right)$ then $L_{1,2}:=S_{1} \cup S_{2}$ because $S_{1} \cup S_{2}$ surely do not interact neither positively nor negatively and we may and want to pursue both goals.

In this way for every pair of goals $g_{i}$ and $g_{j}, \mathrm{i}, \mathrm{j} \in$ $\{1, \ldots, n\}$ decision sets are aggregated. The importance of goals is expressed by the so called priorities. A priority of a goal $g_{i}$ is a real number $\mathrm{P}_{i} \in[0,1]$. The comparison of the priorities is modeled based on the linear ordering of the real interval $[0,1]$. The statements like $g_{i}$ slightly more important than $g_{j}$ are defined as linguistic labels that simply express the extent of the difference between $\mathrm{P}_{i}$ and $\mathrm{P}_{j}$.

\subsection{Multiple goal aggregation as final aggregation based on the local decision sets}

The next step of the aggregation process is the final aggregation. The final aggregation is performed based on a sorting procedure of all local decision sets $\mathrm{Li}, \mathrm{j}$. Again the priority information is used to build a semilinear hierarchy of the local decision sets by sorting them. The sorting process sorts the local decision sets with respect to the priorities of the goals. Subsequently an intersection set of all local decision sets is built. If this intersection set is empty then the intersection of all local decision sets except the last one in the hierarchy is built. If the resulting intersection set again is empty then the second last local decision set is excluded from the intersection process. This process iterates until the intersection is not empty (or more generally speaking until its fuzzy cardinality is big enough with respect to a given threshold). The first nonempty intersection in the iteration process is the final decision set and the membership values of this set give a ranking of the decision alternatives that is the result of the aggregation process (for more details see [4]).

The result is a kind of maximally consistent subset of goals (or criteria). Alternatively the concept of local decision sets could be generalized. However, then the computational complexity of the calculation of the decision sets would increase.

\subsection{Complexity results of the aggregation process}

As already discussed for instance in [6] the complexity of the aggregation process is 
$\mathrm{O}\left((\operatorname{card}(\mathrm{A}))^{2} *(\operatorname{card}(\mathrm{G}))^{2}\right)$ where "card" means the cardinality of a set and the complexity of the information required for the description of both the positive and the negative impact functions is $\mathrm{O}(\operatorname{card}(\mathrm{A}) * \operatorname{card}(\mathrm{G}))$.

\section{Application of the aggregation process to optimi- zation and the complexity of the optimization}

Let us now consider the set $\mathrm{A}^{*}$ defined as extension of the set $\mathrm{A}$ as used in definition Def. 1a). A* is defined as set of all concatenations over A. If $\mathrm{A}^{*}$ is the optimization space of a given optimization problem then the optimization over $\mathrm{A}^{*}$ can be understood as the process of calculation of a sequence of single decisions over A. Each such sequence of $k$ single decisions (let us call it $\mathrm{SEQD} k)$ generates an optimization decision $o_{d}=\left(o_{d l}, .\right.$. , $\left.o_{d k}\right) \in \mathrm{A}^{*}, o_{d i} \in \mathrm{A}$ as result of SEQD $k$.

In many real world optimization problems a solution has the structure of an $o_{d}=\left(o_{d l}, . ., o_{d k}\right)$ as described above. In such cases SEQD $k$ can be considered as an iterating heuristic algorithm that by calculating a sequence of $\mathrm{k}$ single decisions generates an optimization decision and therefore a solution to the given optimization problem. If the optimization goals are defined in the sense of positive and negative impact functions according to definition Def. $1 a$ ) then every single decision is a multiple goal aggregation over $\mathrm{A}$ as described in sections 2.3 and 2.4 and each $\mathrm{SEQD} k$ generates a possible solution to a multiple goal optimization problem with respect to the goal set $\mathrm{G}$.

The quality of the generated solution depends on whether or not the local decisions are good in the sense of the (global) goals of the optimization problem defined over $\mathrm{A}^{*}$. Of course, it is not proved that a SEQD $k$ generates a general optimal solution. But in case that the goals $\mathrm{G}$ over A are defined in a way that they well express the goals over $\mathrm{A}^{*}$ then $\mathrm{SEQD} k$ can generate a good optimization solution and both the positive and the negative impact functions can be aggregated over $o_{d}$ $=\left(o_{d l}, . ., o_{d k}\right)$ and provide for an evaluation of the quality of the solution $o_{d}$ using DMIG itself for the aggregated evaluation.

In real world problems the goal set $\mathrm{G}$ usually can be understood as derived from key performance indicators (KPIs) that describe the quality of the business process to be optimized. Hence there is a direct correlation between $\mathrm{G}$ and the KPIs of the process to be optimized. Therefore the quality of a solution is not too difficult to be evaluated in terms of the KPIs and therefore in terms of $\mathrm{G}$.

Please note as well that in real world optimization problems the dimension of $k$ may be huge but is limited and usually can be estimated by upper boundaries. The reason for the limitation is that many relevant optimization problems are related to business processes for which the optimization is running against limited capacities of resources. In many relevant cases $k$ is calculated before the optimization starts and is therefore a constant. As result of this the $\mathrm{O}$ calculus complexity of a particular SEQD $k$ can be estimated as $\mathrm{O}(k) * \mathrm{O}(\operatorname{card}(\mathrm{A})$ $* \operatorname{card}(\mathrm{G}))=\mathrm{O}(\operatorname{card}(\mathrm{A}) * \operatorname{card}(\mathrm{G}))$ being still efficient.

\section{The problem of workforce management as opti- mization of key performance indicators}

In the process of workforce management there is a set of tasks TS. For each task $t \in \mathrm{TS}$ there is a first possible point of time when working on the task may be started (called ASAP for 'as soon as possible') and the latest point of time when the task has to be finished (ALAP for 'as late as possible'). The workforce consists of a set of teams TM of employees who have qualification profiles QP. Furthermore the teams are physically located in a service area at its locations L. Each team is initially located at its initial location IL more or less close to the places where the tasks have to be done. Each team possesses the possibility to move in the service area (for instance using vehicles). After a task $t$ has been done every team $t m \in$ TM may move from its initial location IL (for instance the place where a team $t$ lives which is a kind of home location HL) to the place of the first task and then to the next task and then to the next and so on.

The possibility to work on a task is restricted by a set of different conditions. One condition is the capacity limitation $C-L$ of each team. The capacity may be limited for instance by the time that a team disposes of. Another limitation is that there may be a maximum movement distance $M-D$. Another one is that only teams with particular qualifications $P-Q$ may work on particular tasks with the limitation $T-Q$. There are several additional limitations. The whole work may be organized in working periods PP, for instance days, being parts of a planning horizon $\mathrm{PH}$, for instance one week or one month. Each task $t$ has to be done in time I-T. 'In time' means that each task $t$ has to be done within the ASAP-ALAP interval and within the planning horizon PH. Each task $t$ may consist of subtasks which may be done in different planning periods PP so that a task may take more than one PP. Every PP may be considered as a limited time sub-horizon and may be associated with the limitations $C-L, M-D, P-Q, T-Q$ etc.

The optimization problem is defined as follows: Attach as many tasks as possible to a number of teams as small as possible in a way that all ASAP-ALAP conditions hold and all other limitations are not violated but exploited to the maximum possible extent. The more the limitations $C-L, M-D, P-Q, T-Q$ etc. are exploited, the higher the number $N-T$ of tasks $t \in \mathrm{TS}$ that are attached to teams $t m \in \mathrm{TM}$ and the smaller the number $N$-TM of teams needed, the better the quality of the optimization result and the better the performance of the whole workforce. In fact the performance exploitation of the limitations $C-L, M-D, P-Q, T-Q$ etc. and the extent to which $N-T$ is maximized and $N-T M$ is minimized are key performance indicators (KPIs) of the workforce management problem. From the point of view of the optimization problem the KPIs described above are the optimization goals.

The decision space of the optimization consists of all possible assignments of tasks $t \in \mathrm{TS}$ to teams $t m \in \mathrm{TM}$ in the planning horizon $\mathrm{PH}$ within the planning periods PP.

Many of the KPIs to be optimized are partly conflicting and therefore negatively correlated. For instance the 
qualification KPIs $P-Q$ and both the movement distance KPIs $M-D$ and the capacity KPIs $C-L$ are conflictive. Also the movement distance KPI $M-D$ and the KPI $I-T$ that expresses that the tasks are done in time are conflictive. On the other hand the minimization of $M-D$ and the KPI that expresses that the teams shall perform tasks close to their initial location $I-L$ help each other and therefore correlate positively. There are some other positively correlated KPI goals, of course.

A more detailed discussion of the optimization goals and of the nature of workforce management as real world optimization problem is given in [9].

\section{KPI-oriented optimization for workforce man- agement using DMIG}

The decision space of the workforce management optimization consists of all possible assignments of tasks $t \in \mathrm{TS}$ to teams $t m \in \mathrm{TM}$ in the planning horizon $\mathrm{PH}$ within the planning periods PP. This means that the decision space is defined as TS $\times$ TM. Therefore we have $k=\operatorname{card}(\mathrm{TS}) * \operatorname{card}(\mathrm{TM})$ and consequently an efficient heuristic SEQD $k$ when we apply the optimization concept based on DMIG as described in section 3 .

In order to illustrate this let us consider as example the KPI expressing the exploitation of the capacity limitation $C$ - $L$ and its modeling as optimization goal using DMIG as follows.

Let aip: $=\left(t_{i p}, t_{m i q}\right) \in \mathrm{TS} \times \mathrm{TM}:=\mathrm{A}$ (for A see definition $1 a$ )) be the decision alternative to attach the task $t p$ to the team $t m_{q}$ in the iteration step $i$ of $\operatorname{SEQD} k, 1 \leq i \leq k$, $i, k \in \mathrm{N}$.

In order to evaluate whether or not aip is a good decision in the iteration step $i$ we estimate that in case that $C-L$ is still very far from the limit the evaluation shall be highly positive (hp). In case that it is yet significantly over the limit then the evaluation shall be highly negative (hn). Close to the limit the evaluation is positive with a rather small value (sp). Close behind the limit the evaluation is rather small negative (sn). Please note that hp, sp, hn, sn are some fuzzy values the definition of which is omitted here without losing generality of the concept.

With such an interpretation of the capacity exploitation KPI we are able to evaluate $C$ - $L$ by applying both the positive and the negative impact functions as defined in Def $1 a$ ) and assign to the corresponding $\delta$ values in $\mathrm{S}_{C-L}$ and $\mathrm{D}_{C-L}$ appropriate membership values for hp, sp and hn, sp etc.

In this way $C$ - $L$ is interpreted as a goal in the sense of DMIG. Of course, all relevant KPIs and therefore all limitations $M-D, P-Q, T-Q$ etc. now can be interpreted in a similar way according to the semantic of the KPIs by defining the corresponding $\mathrm{S}_{M-D}, \mathrm{D}_{M-D}, \mathrm{~S}_{P-Q}, \mathrm{D}_{P-Q}, \mathrm{~S}_{T-}$ $Q, \mathrm{D}_{T-Q}$, etc.

Having done this now DMIG is applied for the iteration steps $i$ of SEQD $k 1 \leq i \leq k, i, k \in \mathrm{N}$ and the decision $a i=\left(t_{i}, t m_{i}\right)$ is made by selecting it out of the set A of decision alternatives $a i p=\left(t_{i p}, t_{i q}\right) \in \mathrm{TS} \times \mathrm{TM}$. The result for the $i^{\text {th }}$ iteration step is the $i^{\text {th }}$ component of the total solution $o_{d}=\left(o_{d l}, \ldots a i=(t i, t m i) \quad \ldots, o_{d k}\right) \in \mathrm{A}^{*}$
SEQD $k$ terminates if a decision is made for all $i$, $1 \leq i \leq k$ or if the KPI limitations are exceeded.

Please note that $k$ is an upper boundary for the number of iterations. In practical cases the number of iteration may be significantly lower than $k$ for instance by using information on existing constraints that reduce the optimization space.

Please also note that there are some similarities between the concept of KPI limitations and the concept of fuzzy valued constraints [1]. However, in contrast to the latter in KPI oriented optimization there is no fixed hierarchy on the KPIs. Many of the KPIs may be equally or similarly conflicting. Therefore in general there is no unique most violated constraint, for instance.

\section{Some remarks on the practical results}

The concept of the DMIG based optimization algorithm presented in the previous sections has been applied to a real world resource planning and optimization problem as part of a workforce management software system in the field of maintenance and fault clearance in electrical grids.

The concept helped to integrate an automated optimization into the workforce management software system. Although people still remain the heart of the workforce management the optimization supports them in many different ways.

Because of the multi-goal nature of the optimization the system is capable to better match the workforce teams with the appropriate qualification. It also helps to better analyze events in the grid and to plan the workforce.

Already the specification of the relevant optimization goals helped to detect conflicts in the processes and provided for a more effective workforce management [9].

\section{Conclusions}

In this paper we have described how an optimization model based on the evaluation of relationships between decision goals helps to solve a relevant real world resource planning problem in an efficient way.

The core of the model is a decision making concept based on fuzzy interactions between decision goals DMIG. It is shown how DMIG can be used to implement iterative heuristics that are oriented on key performance indicators (KPIs) that are interpreted as optimization goals. It is also shown how the optimization model has been applied to the so called workforce management problem.

In workforce management the problem is how to attach tasks to resources in a way that all tasks are finished in time, the capacities of the resources are optimally used and a set of additional limitations are optimally exploited. In this sense workforce management is representative for many real world resource planning problems.

The positive real world results achieved with the optimization model underline its relevance. 


\section{References}

[1] M.C. Cooper, Reduction operations in fuzzy or valued constraint satisfaction, In: Fuzzy Sets and Systems, Vol.134, 311-342, 2003.

[2] D. Dubois and H. Prade, On the use of aggregation operations in information fusion processes, In: Fuzzy Sets and Systems, Vol.142, 143-161, 2004.

[3] R. Felix, Relationships between goals in multiple attribute decision making. In: Fuzzy Sets and Systems, Vol.67, 47-52, 1994.

[4] R. Felix, Decision-making with interacting goals. In: Handbook of Fuzzy Computation, Ruspini, E., Bonissone, P.P, Pedrycz, W. (Eds.), IOP Publishing Ltd, 1998.

[5] R. Felix, Real World Applications of a Fuzzy Decision Model Based on Relationships between Goals (DMRG). In: Forging the New Frontiers, Fuzzy Pioneers I (1965-2005), Springer-Verlag in the series Studies in Fuzziness and Soft Computing, 2007.

[6] R. Felix, Multicriterial Decision Making (MCDM): Management of Aggregation Complexity through Fuzzy Interactions between Goals or Criteria. In: Proceedings of the $12^{\text {th }}$ International IPMU Conference, Málaga, Spain, 2008.

[7] R. Felix, Multi-Goal Aggregation of Reduced Preference Relations Based on Fuzzy Interactions between Decision Goals. In: Proceedings of the IFSA World Congress, Lisbon, Portugal, 2009.

[8] R. Felix, Aggregation of Partly Inconsistent Preference Information. In: Proceedings of the 13th International IPMU Conference, Dortmund, Germany, $178 \mathrm{ff}, 2010$.

[9] E. Jaeker, R. Felix, D. Seifert and T. Epler, MultiCriteria Optimization in Workforce Management. In: Proceedings of the $21^{\text {st }}$ International Conference on Electricity Distribution, Frankfurt, Germany, Paper 0362, 2011.

[10] F. Modave, D. Dubois, M. Grabisch and H. Prade, A Choquet integral representation in multicriteria decision making. In: AAAI Fall Symposium, Boston, Ma, 1997.

[11]F. Modave and M. Grabisch, Preference representation by a Choquet integral: Commensurability hypothesis. In: Proceedings of the $7^{\text {th }}$ International IPMU Conference, Paris, France, 164-171, 1998.

[12] T.L. Saaty, The Analytic Hierarchy Process, Mc Graw-Hill, 1980.

[13] V. Torra, Weighted OWA operators for synthesis of information. In: Proceedings of the fifth IEEE International Conference on Fuzzy Systems, New Orleans, USA, 966-971, 1996.

[14] R.R. Yager, Families of OWA operators. In: Fuzzy Sets and Systems, Vol. 59, 125-148, 1993. 